\title{
The Role of Parvalbumin, Sarcoplasmatic Reticulum Calcium Pump Rate, Rates of Cross-Bridge Dynamics, and Ryanodine Receptor Calcium Current on Peripheral Muscle Fatigue: A Simulation Study
}

\author{
Oliver Röhrle, ${ }^{1,2}$ Verena Neumann, ${ }^{1}$ and Thomas Heidlauf ${ }^{1,2}$ \\ ${ }^{1}$ Institute of Applied Mechanics (CE), University of Stuttgart, Stuttgart, Germany \\ ${ }^{2}$ Stuttgart Research Centre for Simulation Technology (SimTech), University of Stuttgart, Stuttgart, Germany \\ Correspondence should be addressed to Oliver Röhrle; roehrle@simtech.uni-stuttgart.de
}

Received 15 April 2016; Revised 18 July 2016; Accepted 21 August 2016

Academic Editor: Thierry Busso

Copyright (C) 2016 Oliver Röhrle et al. This is an open access article distributed under the Creative Commons Attribution License, which permits unrestricted use, distribution, and reproduction in any medium, provided the original work is properly cited.

\begin{abstract}
A biophysical model of the excitation-contraction pathway, which has previously been validated for slow-twitch and fast-twitch skeletal muscles, is employed to investigate key biophysical processes leading to peripheral muscle fatigue. Special emphasis hereby is on investigating how the model's original parameter sets can be interpolated such that realistic behaviour with respect to contraction time and fatigue progression can be obtained for a continuous distribution of the model's parameters across the muscle units, as found for the functional properties of muscles. The parameters are divided into 5 groups describing (i) the sarcoplasmatic reticulum calcium pump rate, (ii) the cross-bridge dynamics rates, (iii) the ryanodine receptor calcium current, (iv) the rates of binding of magnesium and calcium ions to parvalbumin and corresponding dissociations, and (v) the remaining processes. The simulations reveal that the first two parameter groups are sensitive to contraction time but not fatigue, the third parameter group affects both considered properties, and the fourth parameter group is only sensitive to fatigue progression. Hence, within the scope of the underlying model, further experimental studies should investigate parvalbumin dynamics and the ryanodine receptor calcium current to enhance the understanding of peripheral muscle fatigue.
\end{abstract}

\section{Introduction}

The motor units of a skeletal muscle range from the smallest motor unit (the motor neuron with the lowest excitation threshold innervating the muscle unit with the fewest and most slowly contracting muscle fibres) to the largest motor unit (the largest motor neuron innervating the muscle unit with the most and fastest contracting muscle fibres) [1]. Sustained muscle contractions at moderate to high force levels lead to fatigue and subsequently to a drop in exerted muscle force. Muscle fatigue can hereby be divided into central and peripheral fatigue [2]. Central fatigue refers to changes in the motor neuron activation pattern leading to a decrease in active force. Peripheral fatigue can be assigned to changes in the pathway from electrical excitation to contraction within the muscle fibres, which varies considerably among the different muscle units of a muscle [3]. For example, changes in the shape and amplitude of the action potential during sustained contractions have an influence on peripheral fatigue $[4,5]$. The action potential is influenced by intracellular and extracellular sodium $\left(\mathrm{Na}^{+}\right)$and potassium $\left(\mathrm{K}^{+}\right)$concentrations as well as by membrane resistance and capacitance. Furthermore, accumulation of $\mathrm{K}^{+}$and depletion of $\mathrm{Na}^{+}$ions in the $\mathrm{t}$-tubule system contribute to fatigue. Due to the small volume of the $t$-tubules, however, the exact concentration changes are difficult to determine experimentally. Other aspects, like the role of $\mathrm{Ca}^{2+}$, are undisputed. Failing to release $\mathrm{Ca}^{2+}$ from the sarcoplasmic reticulum (SR) causes fatigue. The $\mathrm{Ca}^{2+}$ concentration in the myoplasm is influenced by the ryanodine receptor (RyR) $\mathrm{Ca}^{2+}$ currents, the SR $\mathrm{Ca}^{2+}$-pump, and several $\mathrm{Ca}^{2+}$ buffers in the cell, such as parvalbumin, calmodulin, and ATP [6]. For repeated muscle contractions, the SR $\mathrm{Ca}^{2+}$-pumping and cross-bridge 
cycling increase the concentration of inorganic phosphate. Experiments suggest that high phosphate levels also play a key role in muscle fatigue.

The differences in the underlying biophysical processes of the different muscle units lead to variations in physiological behaviour such as in an alteration of the contraction speed, intracellular calcium $\left(\mathrm{Ca}^{2+}\right)$ handling, or fatigue resistance [7]. While the differences in physiological behaviour are used to classify different muscle units, the expression of a part of the myosin molecules, the myosin heavy chain (MHC), is the most established classification for muscle units. The isoform of the MHC influences the rate of cross-bridge cycling and thus the maximal shortening velocity [8]. Based on the MHC classification, the major fibre types are types I, IIa, IIx, and IIb. This classification corresponds to the one developed in [3], distinguishing slow-contracting fibres (type S), fast-contracting fatigue-resistant fibres (type FR), and fastcontracting fatigable fibres (type FF) by a certain stimulation protocol (cf. [9]). This classification into discrete classes, however, does not apply to human motor units $[10,11]$. There, a rather continuous variation from one extreme to another is observed for many functional properties [10, 12]. In fact, functional muscle properties such as contraction time, maximum shortening velocity, or the activity of the mitochondrial enzymes such as succinate dehydrogenase $(\mathrm{SDH})$ show large and continuous ranges of variability across the motor units of human skeletal muscles [13].

The continuous variation of physiological properties within muscle units and the large number of different physiological quantities involved within the fatigue process challenge experimentalists to identify the key mechanisms contributing to fatigue. A detailed biochemical in silico model of force generation and fatigue in skeletal muscle can provide an analytical tool to analyse the sensitivity of muscle fatigue due to changes in individual processes or ion concentrations. Therefore, such a computational model could provide insights on the quantities playing a key role in muscle fatigue and hence support experimentalists in interpreting their findings.

As far as in silico models are concerned, there exists a large number of skeletal muscle models. However, many of these models either focus only on small parts of the excitationcontraction coupling (ECC) or phenomenologically describe the respective relationships and are therefore of less use in further investigating muscle fatigue. An exception is the biophysical model of the ECC published in [14]. This model unifies descriptions of membrane voltage [15], release of $\mathrm{Ca}^{2+}$ from the SR [16], activation of the cross-bridge cycling [17, 18], and the resorption of $\mathrm{Ca}^{2+}$ into the SR. Thus, it models all relevant processes of the ECC in a biophysical manner. The model includes aspects of muscle fatigue by assuming that inorganic phosphate, which is generated during crossbridge cycling, is passively transported into the SR, where it can precipitate with $\mathrm{Ca}^{2+}$. As a consequence, less $\mathrm{Ca}^{2+}$ can be released from the SR and fewer cross-bridges can take part in the contraction yielding less force. Moreover, the HodgkinHuxley-type model of the membrane electrophysiology is capable of describing membrane fatigue by the accumulation of $\mathrm{K}^{+}$and depletion of $\mathrm{Na}^{+}$ions in the t-tubule system and the extracellular space.

In [14], two sets of parameters are provided: one for fasttwitch and one for slow-twitch muscles. Both parameter sets are based on literature values. The fast-twitch parameter set has been validated using experimental data obtained through investigating the effects of different electrical stimulation patterns on force production in mouse extensor digitorum longus (EDL) and the slow-twitch parameter set by doing similar experiments on the mouse soleus muscle. While these parameter sets represent extreme values, data for other muscle units are lacking.

To provide muscle unit specific parameters for investigating the key underlying physiological processes leading to fatigue, this work focuses on investigating different interpolation assumptions leading to individual parameter sets for distinct muscle unit types. Further, a sensitivity analysis is performed to investigate how the interpolated parameters, specifically the parameters associated with parvalbumin, the SR calcium pump rate, the cross-bridge cycling rates, and the RyR calcium current influence peripheral muscle fatigue. Note that it is not the aim of this study to identify an optimal set of parameters to model a specific muscle but rather to identify the parameters that significantly affect the considered functional measures, to investigate the sensitivity of the model to changes in the parameters, and to demonstrate that the approach of parameter interpolation is flexible enough to account for many different muscles.

\section{Methods}

2.1. Model Extension. This work is based on the biophysical model of the excitation-contraction coupling of Shorten et al. [14], which can be freely downloaded from the CellML website (https://www.cellml.org/). For the model, two distinct sets of parameters exist. Due to the fact that mouse EDL muscle consists almost exclusively of type-II fibres [19], the fast-twitch parameter set in [14] can be associated with a fasttwitch muscle unit. Similarly, soleus muscle consists mainly of type-I fibres [20], and hence the slow-twitch parameter set in [14] is associated with a slow-twitch muscle unit. These two sets of parameters are the basis for this work.

In the present work, the behaviour of a small human muscle consisting of 100 muscle units (e.g., a hand muscle, such as the first dorsal interosseous [10]), is investigated. To take into account that contraction times are longer in human than in mouse muscles [11, 14, 21], all parameters associated with cross-bridge cycling have been scaled by $1 / 10$. Note that the rates obtained from this scaling for the slow-twitch version correspond to the parameters originally proposed by Razumova et al. for their cross-bridge dynamics model [18], which is used within [14]. The resulting contraction times of $93.7 \mathrm{~ms}$ and $30.5 \mathrm{~ms}$ for the slow-twitch muscle unit and the fast-twitch muscle unit, respectively, compare well to the values proposed in [11].

One drawback of the Shorten et al. model in the context of the current work, however, is the fact that the binding of calcium and magnesium ions to parvalbumin is modelled in a simplified way. In the model, the binding relies on the 


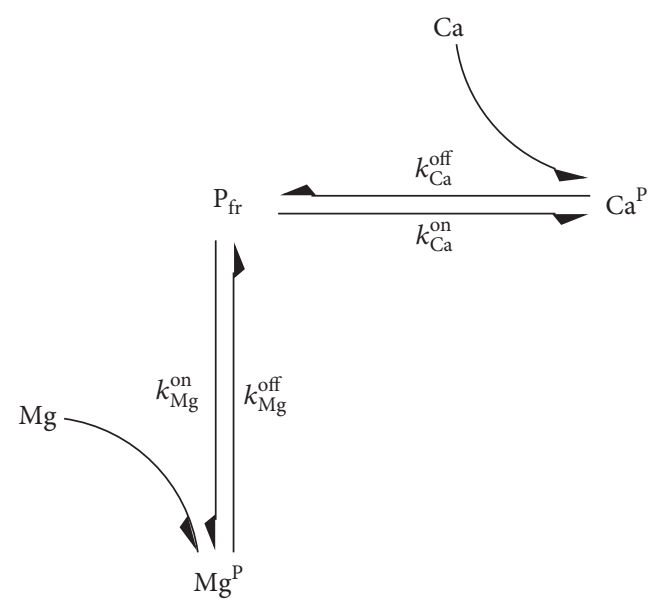

FIGURE 1: Chemical equilibrium model for parvalbumin.

total amount of parvalbumin in the cell. This modelling assumption is sufficient if one intends to consider only typeI and type-II muscle units. In detail, following [22], Shorten et al. consider parvalbumin only in the fast-twitch version of their model, while the parvalbumin binding rates in the slowtwitch version are set to zero. Since the total parvalbumin concentration is not known for intermediate muscle units, the approach needs to be modified.

The parameter representing the total parvalbumin concentration can be omitted if the process is modelled as described in the following. Parvalbumin can bind either to calcium or to magnesium. The respective binding rates are $k_{\mathrm{Ca}}^{\mathrm{on}}$ and $k_{\mathrm{Mg}}^{\mathrm{on}}$; the respective dissociation rates are $k_{\mathrm{Ca}}^{\mathrm{off}}$ and $k_{\mathrm{Mg}}^{\text {off }}$. The reaction kinetics involving the concentration of free parvalbumin, $\mathrm{P}_{\mathrm{fr}}$, are depicted in Figure 1 and are described by

$$
\frac{\mathrm{dP}_{\mathrm{fr}}}{\mathrm{dt}}=-k_{\mathrm{Ca}}^{\mathrm{on}} \mathrm{P}_{\mathrm{fr}} \mathrm{Ca}-k_{\mathrm{Mg}}^{\mathrm{on}} \mathrm{P}_{\mathrm{fr}} \mathrm{Mg}+k_{\mathrm{Ca}}^{\text {off }} \mathrm{Ca}^{\mathrm{P}}+k_{\mathrm{Mg}}^{\text {off }} \mathrm{Mg}^{\mathrm{P}},
$$

where Ca represents the concentration of calcium ions, $\mathrm{Mg}$ is the concentration of magnesium ions, and $\mathrm{Ca}^{\mathrm{P}}$ and $\mathrm{Mg}^{\mathrm{P}}$ denote the parvalbumin bound calcium and the parvalbumin bound magnesium, respectively. Equation (1) is added to the general myoplasm and to the terminal SR myoplasm compartments. The parameter representing the concentration of free parvalbumin is then inserted into the equations of the model, where free parvalbumin is originally modelled by the difference of total parvalbumin concentration (constant) and the sum of the parvalbumin bound ion concentrations. This model extension now allows investigating different parameter sets for intermediate muscle units.

Interpolation of Material Parameter Sets for Intermediate Muscle Units. Currently, there exists no experimental study that could provide for this model a reasonable set of parameters to simulate intermediate muscle units. However, using in silico experiments, parameter sets for intermediate muscle units can be derived from the two extreme parameter sets, that is, from the parameter sets for slow-twitch and fast-twitch muscle units, through interpolation.

In the following, the slowest muscle unit is denoted by $\alpha=0$ and the fastest muscle unit by $\alpha=1$. Further, their corresponding parameter values are denoted by $\mu_{0}$ and $\mu_{1}$, respectively. Following $[10,11]$, this work assumes that the biophysical properties of muscle units are distributed continuously. Hence, the set of parameters for an intermediate muscle unit can be interpolated using a monotone continuous function. Here, the following interpolation functions are considered:

$$
\mu(\alpha, S)=\left(1-\alpha^{p_{i}}\right) \cdot \mu(0, S)+\alpha^{p_{i}} \cdot \mu(1, S),
$$

where $\alpha \in[0,1], p_{i} \in \mathbb{R}^{+}, \mu(\alpha, S)$ is the interpolation of parameter $S$ for intermediate muscle unit $\alpha$, and $p_{i}$ denotes the exponent that characterises the curvature of the function interpolating the two fix points, that is, the polynomial interpolation of the respective slow-twitch $(\alpha=0)$ and fasttwitch $(\alpha=1)$ values, $\mu(0, S)$ and $\mu(1, S)$, respectively, of parameter $S$. Note, for $p_{i}=1$, the respective parameters are interpolated linearly. For $p_{i}>1$ the interpolated values remain for a wider range of $\alpha$ closer to the first value, $\mu(0, S)$, before accelerating to the second value, $\mu(1, S)$. For $p_{i}<1$, the behaviour is just the opposite.

Within the extended model, there are a total of 31 parameters that vary from muscle unit to muscle unit (while 74 of the model's 105 parameters are similar in the slow-twitch and fast-twitch parametrisations). In order to manage this multitude of parameters, all of the 31 parameters are assigned to one of the following 5 groups.

Group 1 contains as only parameter the $\mathrm{Ca}^{2+}$ uptake rate into the SR. This parameter is expected to influence the contraction times. The rate of force generation decreases with increasing rate of the $\mathrm{Ca}^{2+}$-pump.

Group 2 groups the reaction rate constants of the crossbridge cycle. They are expected to change the contraction times [24].

Group 3 contains as only parameter the RyR Ca ${ }^{2+}$ current. Like the $\mathrm{Ca}^{2+}$ uptake rate, the RyR Ca ${ }^{2+}$ current is expected to influence the contraction times as the rate of force generation increases with the rate of $\mathrm{Ca}^{2+}$ release (RyR current).

Group 4 groups parameters that describe the binding to and the dissociation from parvalbumin of $\mathrm{Ca}^{2+}$ and magnesium $\left(\mathrm{Mg}^{2+}\right)$ ions. Parvalbumin is the most important antagonist of troponin and thus influences the activation of the cross-bridge cycling. A change in fatigue characteristics is expected.

Group $R$ contains the rest of the parameters. These parameters are interpolated linearly by default.

Figure 2 depicts a schematic overview of the model with variable parameters. Each number indicates a parameter that has a different value for fast-twitch and slow-twitch muscle units and thus will be interpolated for intermediate muscle units. 


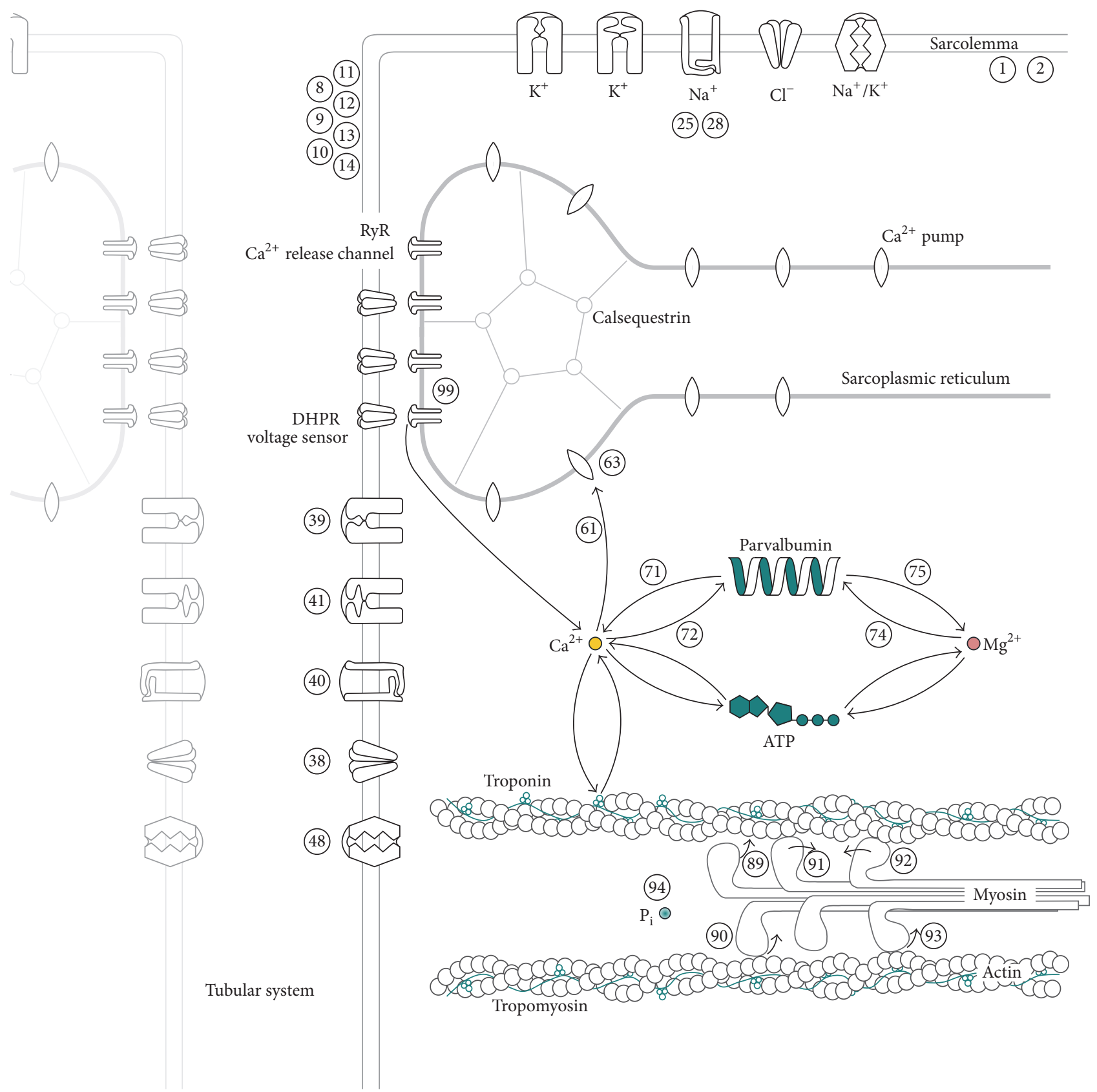

FIgURE 2: Schematic drawing of the cell model with variable parameters. The names of the parameters associated with the numbers within this drawing are given in Table 1.

The grouping of these parameters can be found in Table 1. By choosing for each group an individual interpolation parameter $p_{i}$, the parameters associated with these groups can be interpolated individually.

2.2. Model Verification Approach. After choosing a particular interpolation, that is, choosing for each group in (2) a specific $p_{i}$, the behaviour of the muscle units is compared to experimental data. Based on the data available from the literature, two characteristic force responses are considered for this purpose: (i) the contraction time (also referred to as time to peak) of the single twitch and (ii) fatigue progression.
The contraction time is defined as the duration of time between initiating a contractile response through a stimulus and the maximum twitch force response. Although each muscle has a specific frequency distribution of the muscle unit contraction times, histograms showing experimental data can be described as nearly Gaussian-shaped; see, for example, [13, 25-28].

For a quantitative evaluation of the simulation results, we use as "gold standard" a Gaussian (normal) distribution of the contraction times with $65 \pm 10 \mathrm{~ms}$ (mean \pm standard deviation). This leads to contraction times approximately between $40 \mathrm{~ms}$ and $90 \mathrm{~ms}$ (cf. [11]). 
TABle 1: Parameter groups.

\begin{tabular}{|c|c|c|}
\hline Group & Parameter & Definition \\
\hline 1 & 61 & $\mathrm{SR} \mathrm{Ca}^{2+}$-pump uptake rate \\
\hline \multirow{6}{*}{2} & 89 & Rate of cross-bridge attachment \\
\hline & 90 & $\begin{array}{l}\text { Rate of pre-power stroke cross-bridge } \\
\text { detachment }\end{array}$ \\
\hline & 91 & Forward rate of the power stroke \\
\hline & 92 & Reverse rate of the power stroke \\
\hline & 93 & $\begin{array}{l}\text { Rate of post-power stroke cross-bridge } \\
\text { detachment }\end{array}$ \\
\hline & 94 & $\begin{array}{l}\text { Rate of myoplasmic phosphate } \\
\text { degradation }\end{array}$ \\
\hline 3 & 99 & $\mathrm{RyR} \mathrm{Ca}^{2+}$ current \\
\hline \multirow{2}{*}{4} & 71,72 & $\begin{array}{l}\text { Binding and dissociation rate of } \\
\mathrm{Ca}^{2+} \text {-parvalbumin }\end{array}$ \\
\hline & 74,75 & $\begin{array}{l}\text { Binding and dissociation rate of } \\
\mathrm{Mg}^{2+} \text {-parvalbumin }\end{array}$ \\
\hline \multirow{12}{*}{$R$} & 1 & Membrane capacitance \\
\hline & 2 & $\begin{array}{l}\text { Ratio of t-tubule membrane area to } \\
\text { sarcolemma membrane area }\end{array}$ \\
\hline & 8,9 & t-tubule diffusion time constant \\
\hline & 10 & Fraction of fibre occupied by t-tubules \\
\hline & 11,12 & Interstitial space diffusion time constant \\
\hline & 13,14 & Resting $\mathrm{K}^{+}$and $\mathrm{Na}^{+}$current \\
\hline & 25,28 & Factors for slow inactivation $\mathrm{Na}^{+}$-gating \\
\hline & $38,39,40$ & $\begin{array}{l}\mathrm{Cl}^{-}, \mathrm{K}^{+} \text {, and } \mathrm{Na}^{+} \text {conductance in two } \\
\text { compartment model }\end{array}$ \\
\hline & 41 & Maximum IR conductance \\
\hline & 48 & Maximum $\mathrm{Na}^{+}-\mathrm{K}^{+}$pump activity \\
\hline & 63 & $\mathrm{SR} \mathrm{Ca}^{2+}$ leak constant \\
\hline & 68 & Binding rate of $\mathrm{Ca}^{2+}$-troponin \\
\hline
\end{tabular}

Group 1 contains the $\mathrm{SR} \mathrm{Ca}^{2+}$-pump uptake rate parameter that is associated with force reduction; Group 2 contains the parameters associated with the cross-bridge cycle; Group 3 contains the parameter associated with rate of $\mathrm{Ca}^{2+}$ release from the SR; Group 4 describes parameters associated with the binding to and the dissociation from parvalbumin; Group $R$ contains the remaining parameters that influence the muscle unit type behaviour within the model.

Fatigue progression is the development of the force under sustained tetanic stimulation. In [23], a $30 \mathrm{~s}$ force record for a fast-twitch muscle fibre stimulated at $70 \mathrm{~Hz}$ is presented. Taking [23] as a reference, the force of a type FF muscle unit $(\alpha=1)$ after $5000 \mathrm{~ms}$ of sustained high-frequency stimulation should be about $70 \%$ to $75 \%$ of its maximum value. The force responses of fast fatigable, fast fatigueresistant, and slow muscle units stimulated at $25 \mathrm{~Hz}$ for $1 \mathrm{~s}$, which can be used as additional experimental data for fatigue progression, is presented in [3].

Furthermore, [3] defined a "fatigue index" by the ratio of the "maximum tension produced during the 120th tetanus (i.e., after 2 min of stimulation) to the tension output during the first tetanus in a standard sequence" (tetanic stimulation for $330 \mathrm{~ms}$ at $40 \mathrm{~Hz}$ repeated every second). This definition leads to the fact that slow muscle units without fatigue have a fatigue index of 1 , while fatigable fast muscle units have a fatigue index of 0 . According to [29], over $60 \%$ of the muscle units have a fatigue index greater than 0.75 , that is, showing only little fatigue.

Due to high computational costs, the stimulation sequence in [3] is not simulated for all intermediate muscle units to determine the fatigue index. Instead, the fatigue index is determined from shorter stimulations. Nevertheless, the frequency distribution based on the fatigue index is adapted from the experimental results in [29]; that is, about $65 \%$ of the muscle units shall not show fatigue. Within this work, one assumes that a muscle unit shows no fatigue if the decline in force is less than $10 \%$ after continuously stimulating the muscle unit for $5000 \mathrm{~ms}$ with $100 \mathrm{~Hz}$.

For a quantitative evaluation of the simulation results, we assumed as "gold standard" that the muscle units with $\alpha=\{0,0.5,0.6,0.7\}$ do not show fatigue after a continuous stimulation with $100 \mathrm{~Hz}$ for $5000 \mathrm{~ms}$ [29], and the force of the muscle unit with $\alpha=1$ is at $70 \%$ of its maximum value [23].

In summary, the verification of the suitability of a particular interpolation scheme describing the biophysical behaviour of the muscle units within the extended model is based on a quantitative comparison between the simulation output and the respective experimental data. In detail, for each combination of interpolation coefficients, the relative errors ( 2 norms of the relative differences between the simulation results and the above defined "gold standards") are computed for the contraction times and the fatigue progression. Furthermore, the sensitivity of fatigue to changes in the exponent $p_{i}$ within the interpolation scheme (2) provides the experimentalist with a simulation-based indication on potentially important mechanisms leading to peripheral muscle fatigue.

\section{Results}

First, the parameters of a specific muscle unit are determined through (2) by choosing a particular $\alpha$ (specifies the muscle unit) and by selecting for each parameter group a specific $p_{i}$. The entire set of parameters is obtained for all 100 muscle units by uniformly sampling $\alpha \in[0,1]$. To determine the contraction times for each parameter set and for each muscle unit, a single twitch is simulated. To compare the simulated contraction times with experimental studies, the contraction times are depicted within a histogram containing 15 bins. For the fatigue analysis, the force responses of 5 different muscle units are simulated, that is, for $\alpha=\{0,0.5,0.6,0.7,1\}$, using a stimulation protocol that continuously excites the cellular model at a frequency of $100 \mathrm{~Hz}$. The stimulation starts after $100 \mathrm{~ms}$ and lasts up to $5000 \mathrm{~ms}$.

The starting point for investigating the fatigue behaviour of intermediate muscle units are simulations, in which the parameter sets are determined through a uniform interpolation; that is, all $p_{i}$ are equal. Subsequently, the parameters in Groups 1-4 are varied by choosing within (2) individual exponents for each parameter group; that is, $\mathbf{p}=$ $\left[p_{1}, p_{2}, p_{3}, p_{4}, p_{R}\right]$, where the subindices refer to the group numbering. Unless otherwise stated, the parameters of Group $R$ are linearly interpolated. For each chosen parameter set, the 
TABLE 2: Relative errors in the fatigue progression $\left(E_{f}\right)$ and the contraction time $\left(E_{\mathrm{CT}}\right)$.

\begin{tabular}{lccccccc}
\hline$p_{1}$ & $p_{2}$ & $p_{3}$ & $p_{4}$ & $p_{R}$ & $E_{f}$ & $E_{\mathrm{CT}}$ & Remark \\
\hline 1 & 1 & 1 & 1 & 1 & 0.9548 & 2.0474 & $*$ \\
2 & 2 & 2 & 2 & 2 & 1.0898 & 1.5683 & $*$ \\
5 & 5 & 5 & 5 & 5 & 0.7774 & 2.2112 & $*$ \\
0.5 & 0.5 & 0.5 & 0.5 & 0.5 & 0.8572 & 2.9541 & $*$ \\
1 & 1 & 1 & 1 & 1 & 0.1201 & 0.9114 & \\
0.5 & 1 & 1 & 1 & 1 & 0.1309 & 1.1356 & \\
0.3 & 1 & 1 & 1 & 1 & 0.1364 & 1.3900 & \\
1.2 & 1 & 1 & 1 & 1 & 0.1166 & 0.9038 & \\
0.5 & 5 & 1 & 1 & 1 & 0.3881 & 1.4458 & \\
0.5 & 9 & 1 & 1 & 1 & 0.1273 & 1.9306 & \\
0.5 & 0.5 & 1 & 1 & 1 & 0.1312 & 1.8316 & \\
0.5 & 3 & 1 & 1 & 1 & 0.3911 & 0.9774 & \\
0.5 & 5 & 2 & 1 & 1 & 0.1811 & 0.3267 & \\
0.5 & 5 & 3 & 1 & 1 & 0.2757 & 0.4990 & \\
0.5 & 5 & 4 & 1 & 1 & 0.3990 & 0.8035 & \\
0.5 & 5 & 3 & 1.5 & 1 & 0.1606 & 0.4371 & \\
0.5 & 5 & 3 & 2 & 1 & 0.1012 & 0.3076 & \\
0.5 & 5 & 3 & 4 & 1 & 0.0288 & 0.2484 & \\
\hline & & & & & &
\end{tabular}

$p_{1}-p_{4}$ are the interpolation coefficients for Groups $1-4$, and $p_{R}$ is the interpolation coefficient for the remaining parameters.

${ }^{*}$ Results before scaling the parameters in the parvalbumin group (Group 4) by $1 / 20$. See text for details.

contraction times histogram and selected motor unit force responses at $100 \mathrm{~Hz}$ stimulation frequency are shown.

The relative errors between selected simulation results and the "gold standards" with respect to the contraction times and the fatigue progression are summarized in Table 2. Note that more simulations have been performed, but the chosen selection is sufficient to indicate the sensitivity of the model to changes in the interpolation coefficients. In detail, if relative small changes in an interpolation parameter led to significant changes in the functional measures, we restrained from displaying further data resulting from simulations where larger variations in the interpolation coefficients have been applied. Due to the fact that similar errors can arise from very different distributions, detailed information on the distribution of the contraction times and the fatigue progression are provided in the following (cf. Figures 3-12 and Supplementary Figures 17-38; see Supplementary Material available online at http://dx.doi.org/10.1155/2016/3180205).

Supplementary Figures 17 and 18 show the abovementioned relations for a linear interpolation of all parameters; that is, $\mathbf{p}=[1,1,1,1,1]$. In addition, other uniform interpolations for all parameters are investigated. Figure 19/Figure 20, Figure 21/Figure 22, and Figure 23/Figure 24 within the Supplementary Material show the results for $\mathbf{p}=$ $[2,2,2,2,2], \mathbf{p}=[5,5,5,5,5]$, and $\mathbf{p}=[0.5,0.5,0.5,0.5,0.5]$, respectively. In all these simulations, intermediate muscle units are more affected by fatigue than the fastest muscle unit $(\alpha=1)$. This behaviour does not agree with experimental data. Since the slowest muscle unit $(\alpha=0)$ shows no fatigue and the parameters associated with the binding to and

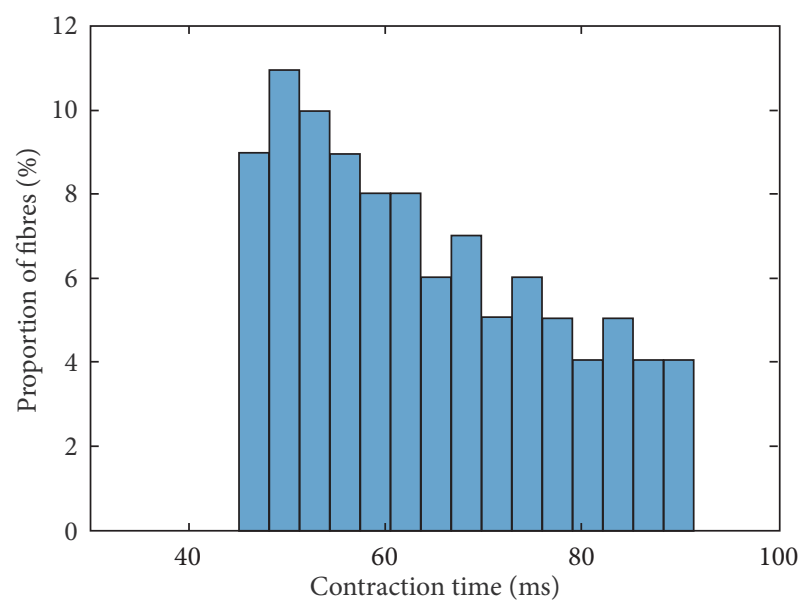

FIgURE 3: Frequency distribution of contraction times based on a linear interpolation of all parameters; that is, $\mathbf{p}=[1,1,1,1,1]$.

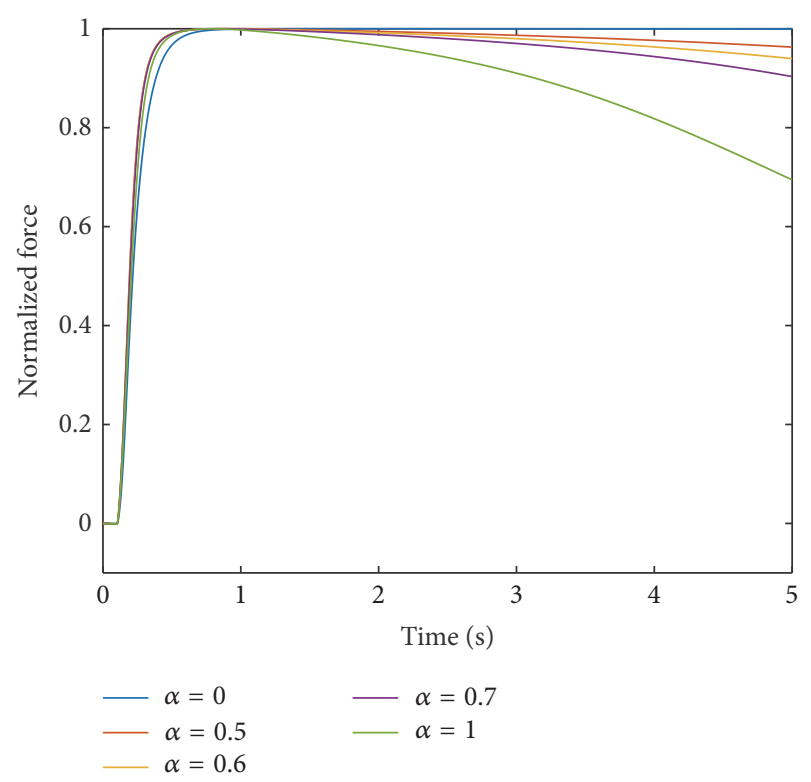

FIGURE 4: Force response at $100 \mathrm{~Hz}$ stimulation for different muscle units obtained through linear interpolation of all parameters; that is, $\mathbf{p}=[1,1,1,1,1]$.

dissociation from parvalbumin are uniformly equal to zero for this muscle unit, it is concluded that the parameters of the parvalbumin group have to be modified first.

3.1. Sensitivity with respect to Parvalbumin Parameters. First, the fast-twitch parameter set is fitted such that the fastest muscle unit $(\alpha=1)$ exhibits $70 \%$ of its maximal force after stimulating the muscle unit for $5000 \mathrm{~ms}$ with a $100 \mathrm{~Hz}$ frequency. This result can only be obtained when the parameters in the parvalbumin group (Group 4 ) are scaled by $1 / 20$. (The parameters of the parvalbumin group for the slow-twitch parameter set are all equal to zero.)

Incorporating this scaling, the contraction times and force responses using a linear interpolation for all parameters, 


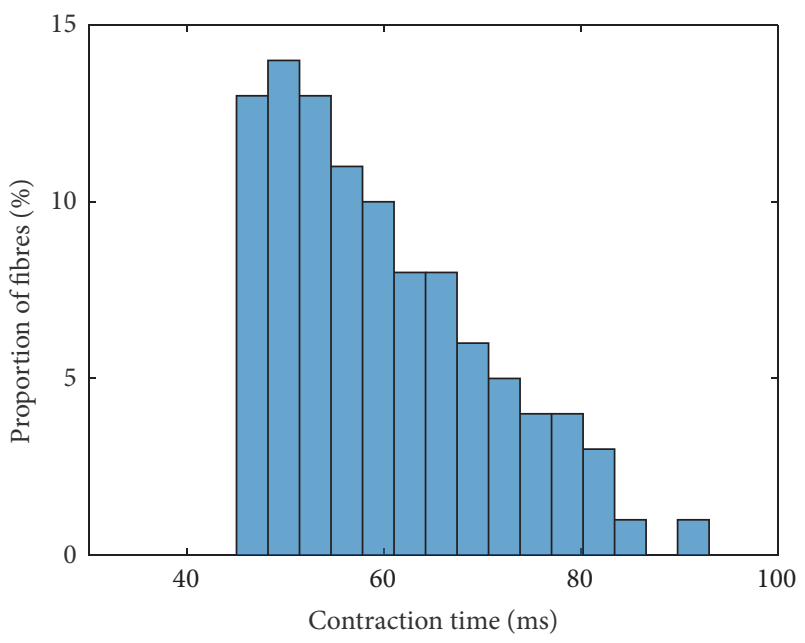

FIGURE 5: Frequency distribution of contraction times based on choosing for interpolation $\mathbf{p}=[0.5,1,1,1,1]$ (modifying the interpolation exponent of the parameter describing the $\mathrm{Ca}^{2+}$-pump uptake rate).

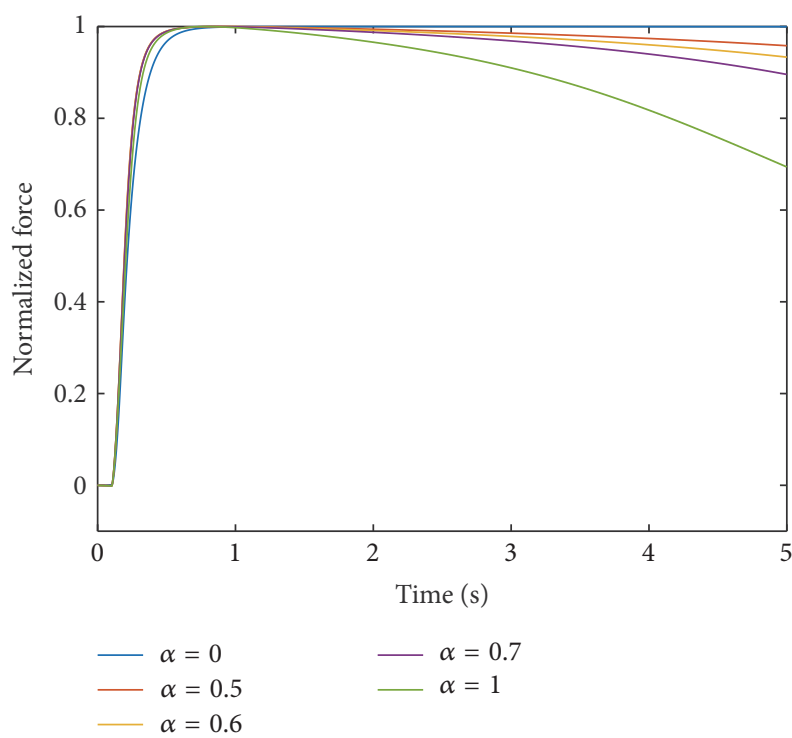

FIGURE 6: Force response at $100 \mathrm{~Hz}$ stimulation for different muscle units obtained by interpolating the parameter sets with $\mathbf{p}=$ $[0.5,1,1,1,1]$ (modifying the interpolation exponent describing the $\mathrm{Ca}^{2+}$-pump uptake rate).

that is, $\mathbf{p}=[1,1,1,1,1]$, are depicted for intermediate muscle units in Figures 3 and 4, respectively.

The contraction times are partially higher compared to the original parvalbumin parameter set (cf. Figures 17 and 18 within the Supplementary Material). They range from $45 \mathrm{~ms}$ to $91 \mathrm{~ms}$. Particularly, the fast-twitch muscle units exhibit reduced contraction times if compared to the original parvalbumin parameter set. As a consequence, the histogram is less steep.

The force of the fastest muscle unit after $5000 \mathrm{~ms}$ is $70 \%$ of its maximum value. All other muscle units show less fatigue than the muscle unit with $\alpha=1$. The muscle units with $\alpha=$

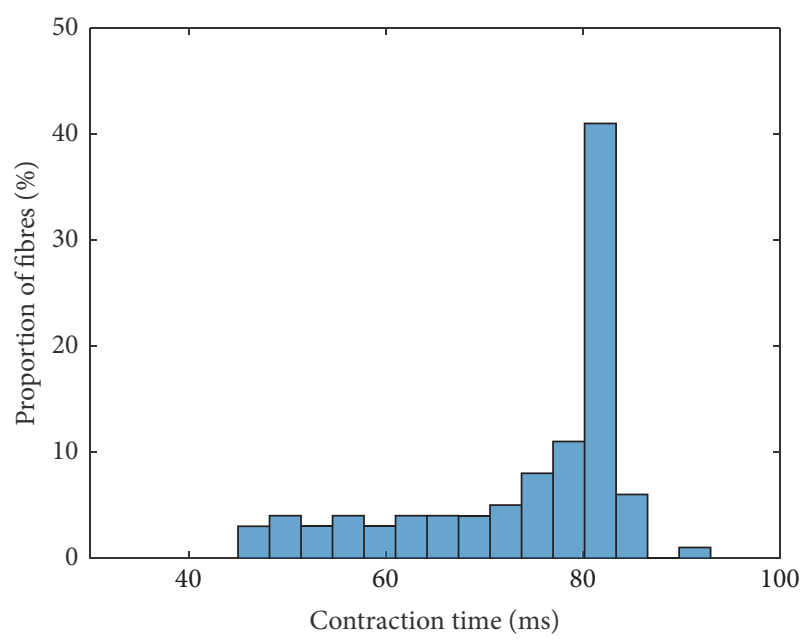

FIgURE 7: Frequency distribution of contraction times based on choosing $\mathbf{p}=[0.5,5,1,1,1]$ for the parameter interpolation.

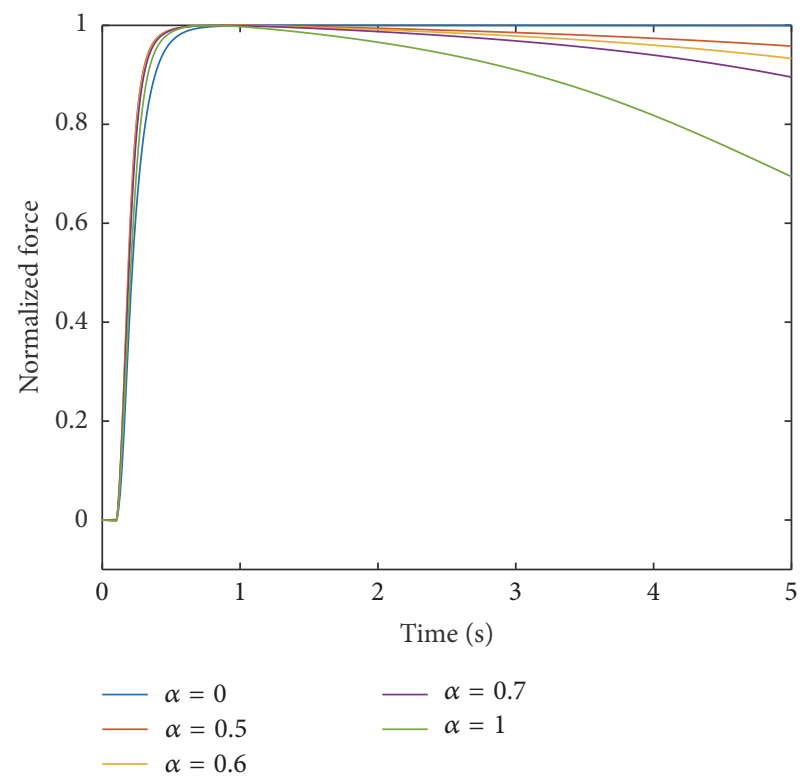

FIGURE 8: Force response at $100 \mathrm{~Hz}$ stimulation for different muscle units obtained by interpolating the parameter sets with $\mathbf{p}=$ $[0.5,5,1,1,1]$.

$0.5,0.6$, and 0.7 are at $96 \%, 94 \%$, and $90 \%$ of their maximum forces, respectively, after $5000 \mathrm{~ms}$.

3.2. Sensitivity with respect to Calcium Pump Uptake Rate. A change in the $\mathrm{Ca}^{2+}$-pump uptake rate into the SR (Group 1) results in a change of the contraction times, especially for slow-twitch muscle units. To demonstrate this, different interpolation exponents $p_{1}$ have been investigated, while a linear interpolation has been chosen for the remaining values of $p_{i}$. Figure 5 shows the results for $\mathbf{p}=[0.5,1,1,1,1]\left(p_{1}=\right.$ 0.5 for the $\mathrm{Ca}^{2+}$-pump uptake rate into the $\mathrm{SR}$ and $p_{2}=p_{3}=$ $\left.p_{4}=p_{R}=1\right)$. Additionally, the results for $p_{1}=0.3$ and $p_{1}=1.2$ are provided in the Supplementary Material (Figures 25 and 27, resp.). 


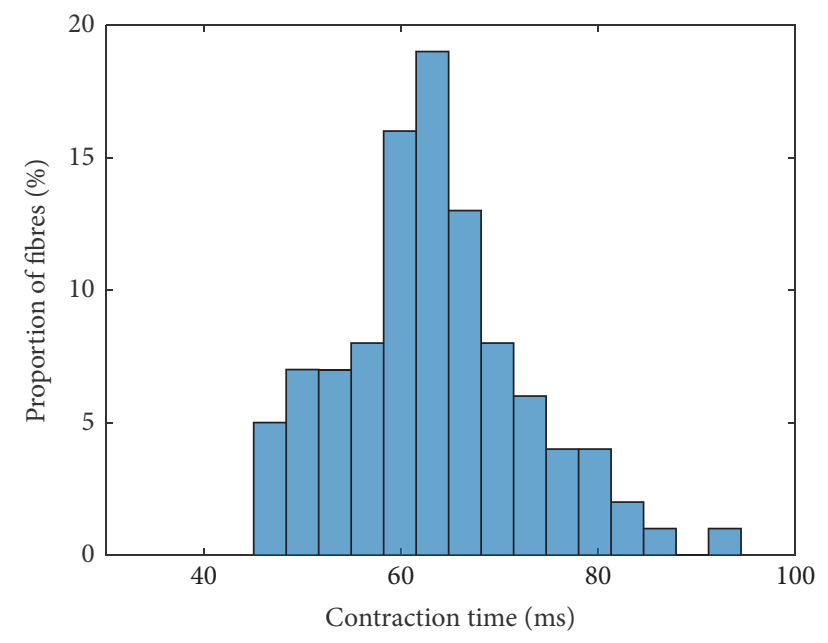

FIGURE 9: Frequency distribution of contraction times based on choosing $\mathbf{p}=[0.5,5,3,1,1]$ for the parameter interpolation.

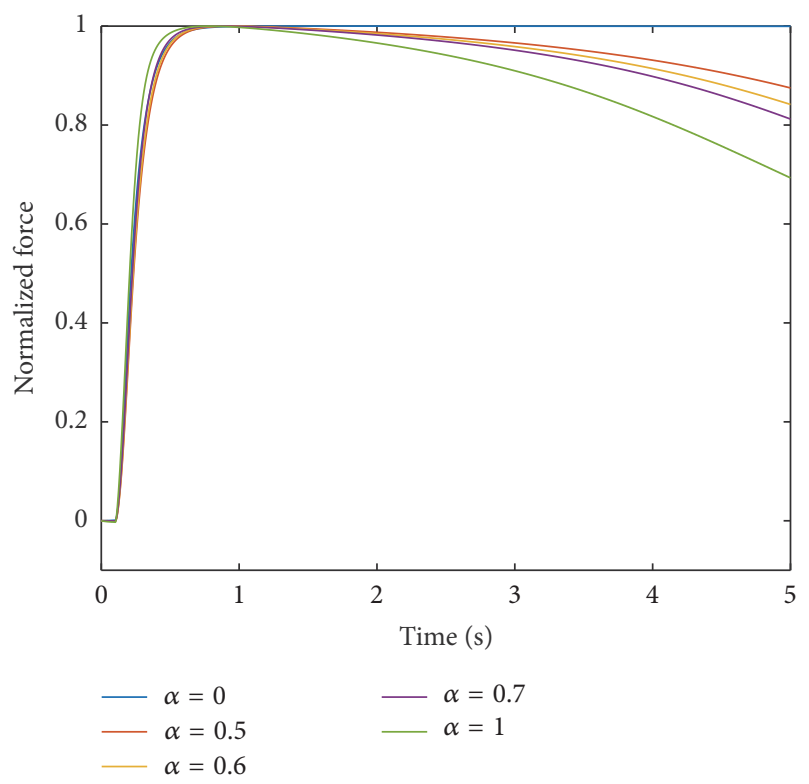

FIGURE 10: Force response at $100 \mathrm{~Hz}$ stimulation for different muscle units obtained by interpolating the parameter sets with $\mathbf{p}=$ $[0.5,5,3,1,1]$.

For $p_{1}=0.5$, the curve showing contraction times versus muscle unit type exhibits a hyperbolic shape and many muscle units have short contraction times. The hyperbolic shape becomes even steeper for $p_{1}=0.3$. For $p_{1}=1.2$, the contraction time decreases almost linearly with increasing values of $\alpha$ and the distribution slightly shifts the contraction times toward shorter values.

Comparing the results on fatigue progression in Figure 6 and Supplementary Figures 26 and 28, one can observe that the $\mathrm{Ca}^{2+}$-pump uptake rate does not influence the fatigue progression by much. For $p_{1}=0.5$ the forces of muscle units with $\alpha=0.5,0.6$, and 0.7 after $5000 \mathrm{~ms}$ of stimulation with frequency $100 \mathrm{~Hz}$ are $96 \%, 93 \%$, and $90 \%$ of their maximal

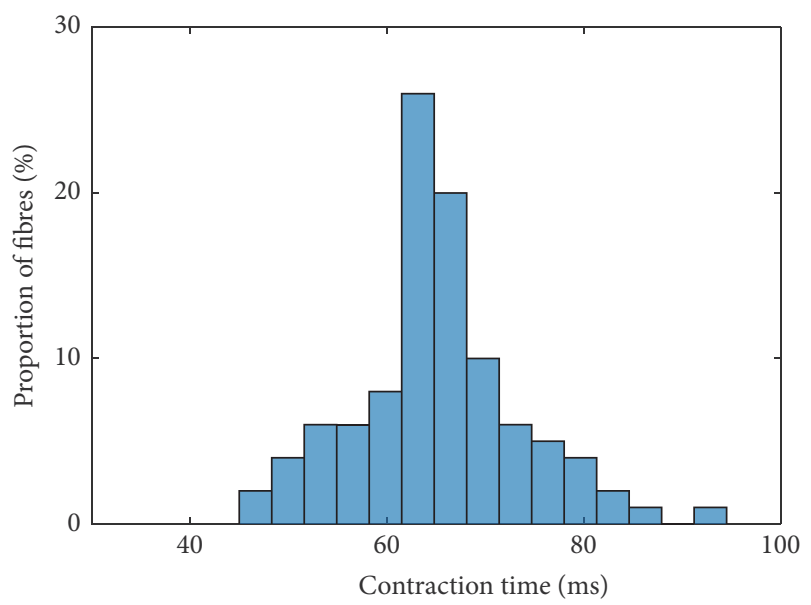

FIGURE 11: Frequency distribution of contraction times based on choosing $\mathbf{p}=[0.5,5,3,4,1]$ for the parameter interpolation.

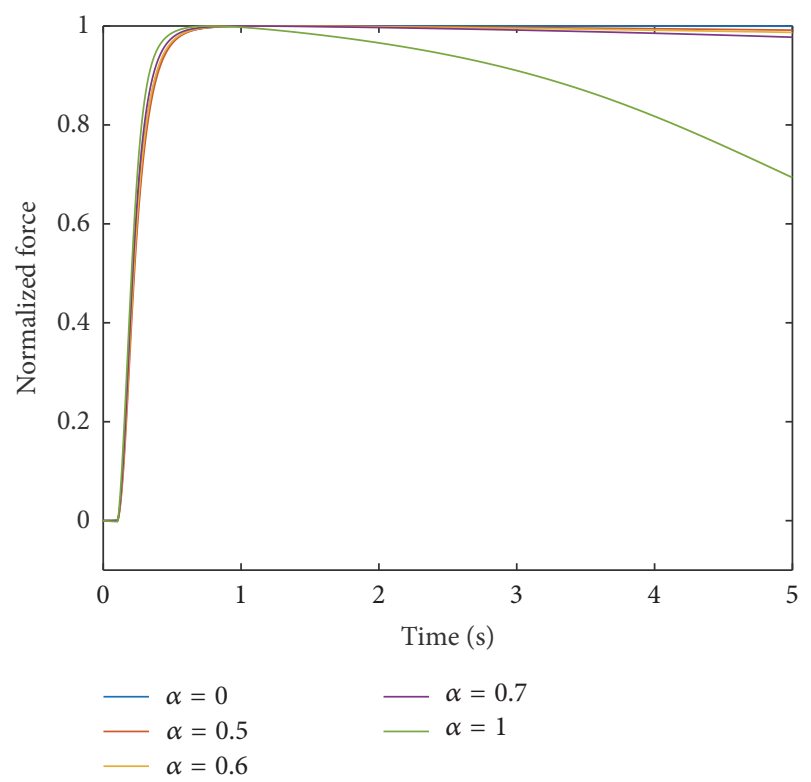

FIGURE 12: Force response at $100 \mathrm{~Hz}$ stimulation for different muscle units obtained by interpolating the parameter sets with $\mathbf{p}=$ $[0.5,5,3,4,1]$.

values, respectively. For $p_{1}=0.3$, the respective forces are $95 \%, 93 \%$, and $90 \%$ of their maximal forces. Moreover, an interpolation with an exponent $p_{1}=1.2$ yields slightly higher remaining forces; that is, the forces of muscle units $\alpha=$ $0.5,0.6$, and 0.7 after $5000 \mathrm{~ms}$ of stimulation with frequency $100 \mathrm{~Hz}$ are $96 \%, 94 \%$, and $91 \%$ of their maximal values, respectively.

3.3. Sensitivity with respect to Cross-Bridge Parameters. Assuming independence of the parameters, the most suitable interpolation of the investigated $\mathrm{Ca}^{2+}$-pump uptake rate parameters provides the basis for further investigations. Next, the sensitivity due to changes in the cross-bridge parameters (Group 2) is analysed. To do so, the model was run using 
the interpolation exponents $p_{2}=0.5, p_{2}=5$, and $p_{2}=$ 9. The interpolation of the parameters of Group 2 affects particularly the contraction times. The contraction times and force responses for different muscle units and interpolation exponent $p_{2}=5$ are shown in Figures 7 and 8, respectively. For $p_{2}=5$, the histogram peaks at contraction times between $80 \mathrm{~ms}$ and $85 \mathrm{~ms}$ (Figure 7). About $41 \%$ of all muscle units fall within this bin. Further results on contraction times and force responses are given for $p_{2}=0.5$ and $p_{2}=9$ in Figure 29/Figure 30 and Figure 31/Figure 32 within the Supplementary Material, respectively. For an interpolation exponent smaller than 1 , one observes again a hyperbolic shape of the contraction times. (The reader is referred to Supplementary Figures 31 and 32, which are associated with $\mathbf{p}=[0.5,0.5,1,1,1]$, for a representative example of a small interpolation exponent $p_{2}$.) For $p_{2}=9$ the peak in the histogram increases, and $61 \%$ of the muscle units exhibit contraction times between $80 \mathrm{~ms}$ and $85 \mathrm{~ms}$ (cf. Supplementary Figures 29 and 30).

Note that fatigue is not affected by adjusting the interpolation parameter $p_{2}$, that is, by changing the interpolation scheme for the parameters associated with Group 2. For all three displayed interpolation exponents $\left(p_{2}=0.5,5\right.$, and 9$)$, the forces of the muscle units with $\alpha=0.5,0.6$, and 0.7 are $96 \%, 94 \%$, and $90 \%$ of their maximum values, respectively, after $5000 \mathrm{~ms}$ stimulation with frequency $100 \mathrm{~Hz}$.

3.4. Sensitivity with respect to the RyR-Channel Rate. For the following simulations, the exponents of the interpolation for $p_{1}, p_{2}$, and $p_{4}$ are adopted from Section 3.3; that is, $\mathbf{p}=\left[0.5,5, p_{3}, 1,1\right]$. Now, the interpolation of the RyRchannel rate (Group 3) is considered, which impacts the contraction times and fatigue. Figure 9/Figure 10, as well as Figure 33/Figure 34 and Figure 35/Figure 36 within the Supplementary Material, depict the histograms of the contraction times and the force responses for $p_{3}=2$ (Figure $33 /$ Figure 34 within the Supplementary Material), $p_{3}=3$ (Figure 9/Figure 10), and $p_{3}=4$ (Figure 35/Figure 36 within the Supplementary Material). For $p_{3}=1$, the histogram shows an extreme accumulation of muscle units at contraction times of approximately $80 \mathrm{~ms}$. By increasing $p_{3}$, the peak of the histogram shifts toward slower muscle units and the extreme accumulation is less pronounced leading to a broader distribution. Specifically, for $p_{3}=2$, the histogram of contraction times peaks at $70 \mathrm{~ms}$. About $55 \%$ of the muscle units show contraction times between $66 \mathrm{~ms}$ and $73 \mathrm{~ms}$ (Figure 9). For $p_{3}=3$, the peak occurs at $63 \mathrm{~ms}$, for $p_{3}=4$ the peak is broader and occurs at about $58 \mathrm{~ms}$. In conclusion, one can claim that the higher the interpolation exponent $p_{3}$ the shorter the most frequent contraction time.

The fatigue progression also changes with different interpolation exponents $p_{3}$. For $p_{3}=2$, the forces of the muscle units with $\alpha=0.5,0.6$, and 0.7 are after $5000 \mathrm{~ms}$ of stimulation with $100 \mathrm{~Hz} 93 \%, 90 \%$, and $86 \%$ of their maximum force, respectively. Further, for the same muscle units, $p_{3}=$ 3 results in remaining forces of $88 \%, 84 \%$, and $81 \%$, while $p_{3}=4$ results in remaining forces of $80 \%, 76 \%$, and $74 \%$, respectively.

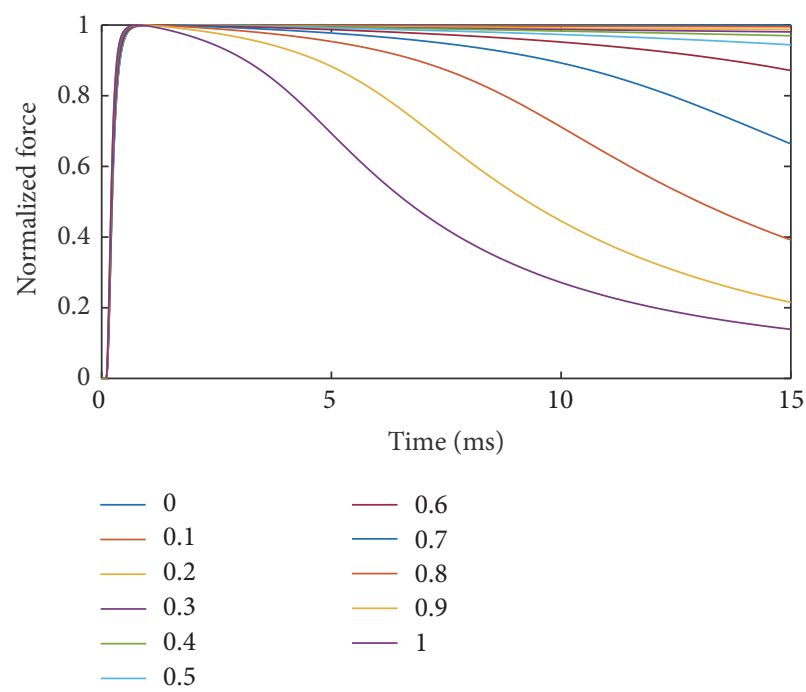

FIGURE 13: Force response at $100 \mathrm{~Hz}$ for different muscle units over a period of $15 \mathrm{~s}$. The parameters for the different muscle units are obtained by interpolating with $\mathbf{p}=[0.5,5,3,4,1]$.

3.5. Determining the Interpolation Exponent For Parvalbumin. As seen in the section that investigated the sensitivity with respect to parvalbumin parameters, the contraction times are not much affected by changes to the parvalbumin parameters (Group 4). This can be seen if, for example, Figure 11 is compared to Supplementary Figure 37. Following this, the interpolation of the parvalbumin parameters is used to fit experimentally determined fatigue characteristics (fatigue occurs for muscle units with $\alpha>0.6$ ). The linear interpolation of the parvalbumin parameters $\left(p_{4}=1\right)$ overestimates the fatigue progression. Hence, interpolation exponents greater than 1 are tested in order to obtain a parameter set for fast fatigable muscle units that is comparable to experimental data. For $p_{4}=1.5$, one obtains for the muscle units with $\alpha=0.5,0.6$, and 0.7 after $5000 \mathrm{~ms}$ of stimulation with $100 \mathrm{~Hz}$ forces of $94 \%, 91 \%$, and $88 \%$ of their maximum values, respectively. Choosing an interpolation exponent of $p_{4}=2$, one obtains for the same muscle units and the same stimulation protocol remaining forces of $97 \%, 95 \%$, and $92 \%$ (Supplementary Figure 38). For $p_{4}=4$ the remaining forces are $99 \%, 99 \%$, and $98 \%$, respectively (cf. Figure 12 ).

Up to now, all simulations were run for $5 \mathrm{~s}$. If the simulations are run for $15 \mathrm{~s}$, one observes that fatigue progresses over time for the muscle units with $\alpha>0.5$. Since this is not observed experimentally, the interpolation exponent for parvalbumin is changed to $p_{4}=4$. Figure 13 presents the results for 11 uniformly distributed muscle units. It can be observed that the fatigue-induced force decline for muscle units with $\alpha \leq 0.5$ is less than $10 \%$. For the remaining muscle units, that is, $\alpha=0.6$ to $\alpha=0.9$, the reduction in force after 15 s covers the spread between the resulting forces of $\alpha=0.5$ and $\alpha=1$.

3.6. Stimulation Patterns of Burke et al. and Lannergren and Westerblad. To verify that the frequency distribution of the fatigue index can be adapted to the distribution of 

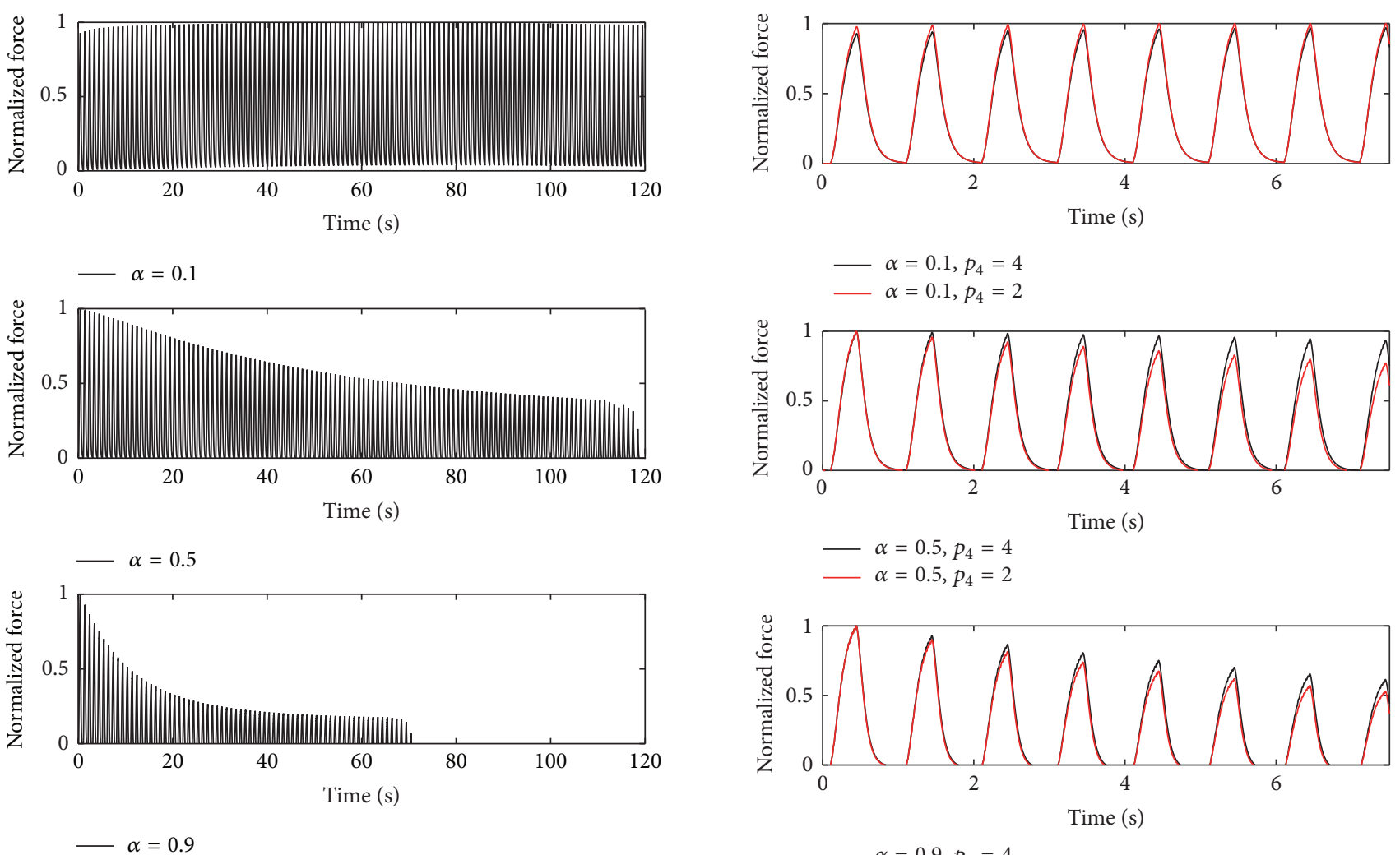

FIGURE 14: Burke et al.s stimulation [3]: the force responses of three muscle unit types ( $\alpha=0.1,0.5$, and 0.9$)$ repetitively stimulated over a period of $120 \mathrm{~s}$.

the short-time fatigue (remaining muscle unit forces after $5000 \mathrm{~ms}$ ) Burke's stimulation pattern [3] is applied to the final parameter set; that is, $\mathbf{p}=[0.5,5,3,4,1]$. Three muscle units $(\alpha=0.1,0.5$, and 0.9$)$ are stimulated for $120 \mathrm{~s}$ at $40 \mathrm{~Hz}$ over a time period of $330 \mathrm{~ms}$. This is repeated every second. (No stimulation is applied in the remaining $670 \mathrm{~ms}$ of each second.) The results are presented in Figures 14 and 15.

In Figure 15, the force response in the first $7.5 \mathrm{~s}$ can be seen for two different parvalbumin interpolation exponents; that is, $p_{4}=2$ and $p_{4}=4$. The solid line shows the force response for $p_{4}=4$; the dashed line is simulated with $p_{2}=2$. For muscle unit types 0.5 and 0.9 , the force declines for $p_{2}=2$ more rapidly than for $p_{4}=4$. Muscle unit type 0.1 shows no fatigue in either case.

The muscle unit with $\alpha=0.1$ shows no fatigue within the interval. Hence, Burke's fatigue index for this muscle unit is 1. The force response of the muscle unit with $\alpha=0.5$ declines approximately linearly until $118 \mathrm{~s}$. The second last force peak is considerably lower than the previous ones. The last force peak is missing entirely. The muscle unit with $\alpha=0.9$ shows an exponential decline in the force response in the first 40 s, before settling at a force of about $20 \%$ of its maximum value. After $68 \mathrm{~s}$ stimulation time, the force decreases rapidly such that no further force peaks can be observed any more after $72 \mathrm{~s}$. Following the nomenclature of [3], this results in a fatigue index of 0 for the muscle units with $\alpha=0.5$ and 0.9 .

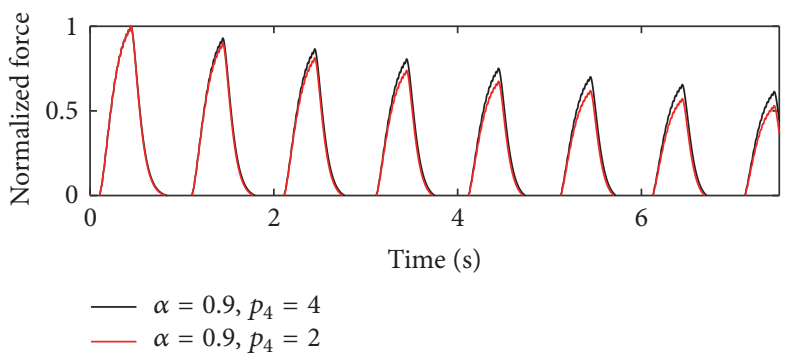

FIGURE 15: Force responses at $40 \mathrm{~Hz}$ stimulation for $220 \mathrm{~ms}$ repeated every second for different muscle units ( $\alpha=0.1,0.5$, and 0.9$)$. Parvalbumin parameters $p_{4}=2$ and $p_{4}=4$ are considered; that is, $\mathbf{p}=[0.5,5,3,2,1]$ and $\mathbf{p}=[0.5,5,3,4,1]$.

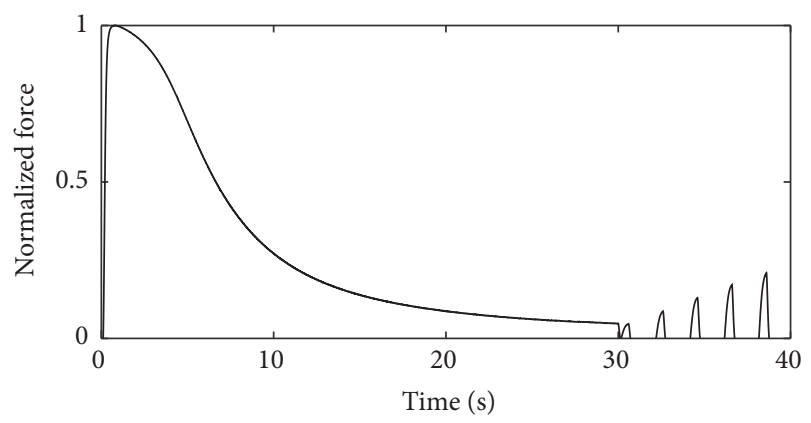

FIGURE 16: Lannergren and Westerblad's stimulation pattern [23]. Force response for a fast-twitch muscle unit $(\alpha=1)$ for a tetanic stimulation followed by a stimulation at $100 \mathrm{~Hz}$ for $500 \mathrm{~ms}$ repeated every two seconds.

Figure 16 shows the force response to a stimulation pattern similar to that presented by Lannergren and Westerblad [23]. The fastest muscle unit $(\alpha=1)$ is first continuously stimulated with a frequency of $100 \mathrm{~Hz}$ for $30 \mathrm{~s}$, followed by a Burke-like stimulation, that is, stimulating every two seconds for $500 \mathrm{~ms}$ with $100 \mathrm{~Hz}$.

The force declines in the first $30 \mathrm{~s}$ to $5 \%$ of the maximum force. The subsequent stimulation with resting time results in 
a regeneration of the force. The peak of the 5 th train of stimuli shows already $20 \%$ of the maximum force. This recovery is qualitatively consistent with experimental data; however, quantitatively, the recovery is not as fast as in experiments.

\section{Discussion}

Before discussing the modelling assumptions and the results in a broader context, the findings of the interpolation study are summarized in the following. Note that for $p_{i}>1$ the interpolated values remain for a wider range of motor units closer to the slow-twitch value, before accelerating to the fasttwitch value. For $p_{i}<1$, the behaviour is just the opposite.

(i) If the binding and dissociation rates to and from parvalbumin are not significantly reduced, intermediate muscle units show more fatigue than the fastest muscle unit (cf. Figure 3 and Supplementary Figures 17, 19, 21, and 23). Such behaviour has not been observed experimentally.

(ii) Increasing (decreasing) the interpolation coefficient for the calcium pump uptake rate, $p_{1}$, leads to a shift toward fast (slow) contraction times (cf. Figure 5 and Supplementary Figures 25 and 27).

(iii) Values of the interpolation coefficient for the reaction rates of the cross-bridge dynamics model, $p_{2}$, greater than one lead to a peak in the contraction times histogram at long contraction times, and the height of the peak increases with $p_{2}$. Values of $p_{2}$ smaller than one yield a shift of the distribution toward slow contraction times (cf. Figure 7 and Supplementary Figures 29 and 31).

(iv) The interpolations of both the calcium pump uptake rate and the cross-bridge dynamics reaction rates hardly affect fatigue.

(v) By increasing the interpolation coefficient of the RyRchannel rate, $p_{3}$, the peak of the contraction times histogram shifts toward slower muscle units and it is less pronounced leading to a broader distribution (cf. Figure 9 and Supplementary Figures 33 and 35). In conclusion, one can claim that the higher the interpolation exponent $p_{3}$, the shorter the most frequent contraction time.

(vi) Increasing the interpolation coefficients of the RyRchannel rate, $p_{3}$, results in faster fatiguing intermediate muscle units.

(vii) Increasing (decreasing) the interpolation coefficient of the parvalbumin parameters, $p_{4}$, leads to weaker (stronger) fatigue progression of intermediate muscle units (cf. Figures 12 and 13 and Supplementary Figure 38).

(viii) Changes in the interpolation coefficient of the parvalbumin parameters, $p_{4}$, do not affect the contraction times (cf. Figure 11 and Supplementary Figure 37).

Although muscle fatigue has been well studied in experiments on whole muscles and single intact and stripped fibres, the cellular processes leading to muscle unit-dependent (peripheral) muscle fatigue are still not entirely understood. Due to the fact that the Shorten et al. model [14] closely reflects the physiological processes leading from electrical excitation to contraction in skeletal muscle fibres and also contains a description of different biochemical processes leading to muscle fatigue, it can be used to generate data that are currently not available from the literature. In the present study, this model has been extended to a description of the different muscle units in a muscle, which are subject to different levels of fatigue. To this end, the parameters of the slow-twitch and fast-twitch versions of the Shorten et al. model [14] have been modified and interpolated using smooth and continuous interpolation functions. While the model is capable of closely matching experimental data, one should mention that, in physiology, some properties might also arise only after a threshold or are grouped into discrete classes. One could certainly consider within this work also step functions and, hence, probably even further improve the results. This, however, leads to further degrees of freedom that make it even harder to investigate and analyse the results.

Continuous distributions of the functional properties (e.g., contraction time, maximum shortening velocity, or the activity of the mitochondrial enzymes such as SDH) across the muscle units of skeletal muscles are well established (cf., e.g., $[10,13]$ and references therein). In contrast, continuous distributions of biophysical parameters, as assumed in this work, have not been described in the experimental literature. Bottinelli and Reggiani [13] distinguish two main mechanisms leading to the heterogeneity among the functional properties of muscle units. First, the heterogeneity can result from different isoforms that exist for many muscle proteins. This does not contradict the assumption of continuous distributions of biophysical parameters, since muscle fibres can coexpress different isoforms. For example, different isoforms of the MHC have been found to coexist in one muscle fibre type [10]. Second, the same gene can be expressed differently, depending on factors such as neural activation, mechanical load, or hormones. For example, the density of SR $\mathrm{Ca}^{2+}$ pumps is much higher in fast-twitch than in slow-twitch muscle fibres [7]. It can be easily imagined that the up- and downregulation of genes yield a continuous distribution of the biophysical properties.

Although closely resembling the physiological processes of the ECC, the model of Shorten et al. [14] does not contain a description of the cell metabolism. In detail, while Shorten et al. model the accumulation of ADP and inorganic phosphate in the myoplasm, there are unlimited amounts of ATP available within the cells. The depletion of ATP and the accumulation of ADP and inorganic phosphate in the myoplasm are known to affect the force generating capability of muscle fibres [6]. Especially for analysing sustained contractions, the model should be extended by a more detailed description of the cell metabolism that accounts, for example, for the ATP generation in the mitochondria and the supply of oxygen and nutrients to the cell. This however is beyond the scope of this work. Including a description of the cell metabolism within the model would affect the fatigue 
progression during sustained contractions as considered in Figures 13, 14, 15, and 16. For now, the predicted fatigue is mainly influenced by the RyR-channel rate and the parvalbumin dynamics (binding and dissociation rates of calcium and magnesium ions to and from parvalbumin). Including the cell metabolism as a third mechanism contributing to fatigue might require a reparametrisation of the RyR-channel rate and the parvalbumin dynamics. In experimental conditions, it is difficult to separate the influence of different factors contributing to fatigue. A biophysical muscle model that includes a description of the cell metabolism could support the experimentalist in this task.

As a model can only be as good as the data to which it is fitted, it is important to identify and focus in experimental studies on those parameters that exhibit the most sensitivity with respect to muscle fatigue. The resulting model produces fast fatigable and slow fatigue-resistant muscle units. What is not well realised yet is the fast fatigue-resistant muscle unit type. This muscle unit type is characterised by a fast contraction (comparable with the fast fatigable muscle unit) and little fatigue. Within the presented model, the fastest muscle unit that shows only little fatigue $(\alpha=0.5)$ contracts in about $60 \mathrm{~ms}$, which is $150 \%$ of the contraction time of the fastest muscle unit. In general, however, the contraction times could be improved by modifying the cross-bridge parameters for fast-twitch muscle units such that the proposed model would exhibit contraction times also below $40 \mathrm{~ms}$. In [11], for example, a contraction time of $30 \mathrm{~ms}$ was assumed for the fastest muscle unit. In the present work, the cross-bridge parameters of Shorten et al. [14] have been scaled by a factor of $1 / 10$ (cf. [18]), to account for slower kinetics and longer muscle fibres in humans compared to mice [21]. This scaling yielded contraction times approximately between $40 \mathrm{~ms}$ and $90 \mathrm{~ms}$. A larger scaling factor, for example, 1/5, probably leads to better results. The aim of this study, however, is not to identify an optimal set of parameters but to investigate the sensitivity of the model to variations in the parameters, in order to reach a conclusion on their importance with respect to muscle fatigue.

Besides the contraction times, the reaction rates of the cross-bridge cycle determine the shape of the single twitch of a muscle fibre. The parameter set suggested by Razumova et al. for their cross-bridge dynamics model [18] (which is used within the model of Shorten et al. [14]) predicts twitch shapes that agree well with experimentally measured ones. A consistent scaling of the entire parameter set of the crossbridge dynamics model of Razumova et al. [18] leads to twitches with different time course but similar shape. For example, Shorten et al. [14] successfully modelled twitches and tetanic contractions of soleus and EDL muscles of mice by multiplying the entire parameter set of Razumova et al. by factors of 10 and 30, respectively. In accordance with this, we grouped together the cross-bridge parameters within one group, such that the resulting model predicts twitch shapes that are scaled versions of the ones predicted by the Razumova et al. model.

Two isoforms, namely, SERCA2 in slow-twitch fibres and SERCA1 in fast-twitch fibres, exist for the SR $\mathrm{Ca}^{2+}$ pump in skeletal muscle, and the density of pumps is much higher in fast-twitch than in slow-twitch fibres (cf. [7]). Following this, the SR $\mathrm{Ca}^{2+}$ pumping rate was treated as an individual parameter group in our model. Moreover, since there exist differences in the kinetics of the RyR between slow-twitch and fast-twitch muscle fibres [13], the $\mathrm{RyR} \mathrm{Ca}^{2+}$ current is also treated as an individual parameter set.

Finally, we investigated the model behaviour for different interpolations of the binding and dissociation rates to and from parvalbumin. Since parvalbumin is only present in fast-twitch fibres [22], the corresponding rates equal zero in the slow-twitch version of the model. We obtained realistic results for an interpolation coefficient of $p_{4}=4$, which means that, for the majority of muscle units, the parvalbumin parameters are similar to the slow-twitch model (i.e., close to zero), and parvalbumin only affects few (fast-twitch) muscle units. This finding corresponds well with the fact that there are typically many slow-twitch and few fast-twitch muscle units in a muscle [11]. Further, for the parameter interpolation, we grouped together the binding and dissociation rates of calcium and magnesium ions to and from parvalbumin into one group. Another possibility would have been to treat each of the binding and dissociation rates independently. This, however, would further increase the model complexity. Moreover, there is no experimental data available to justify a certain choice over another.

The outcome of stimulating the fastest muscle unit with a long-term tetanic stimulation protocol with respect to fatigue fits well the experimental data presented in [23]. The progression of the other muscle units cannot be reviewed as there is no experimental data available. The fatigue of intermediate muscle units was designed such that only $30 \%$ of the muscle units show fatigue. This is in agreement with the experimental data presented in [29]. The parameters, however, could be further improved given further data. In this work, the fatigue of different muscle units was adapted to the frequency distribution of fatigue indices following [3]. To confirm this approach, it was expedient to use a stimulation protocol based on the pattern proposed in [3]. The results for three different muscle unit types ( $\alpha=0.1,0.5$, and 0.9 ) over a period of $120 \mathrm{~s}$ can be seen in Figure 14. The presented simulation shows that the parameters could still be improved by fitting them to new experimental data as they become available. However, it was not the objective of this work to provide a perfect set of parameters to model a specific muscle but rather to reveal the sensitivity of the model response to changes in the parameters.

Applying Lannergren and Westerblad's stimulation pattern [23] to a fast-twitch muscle fibre, the model predicted a slow recovery. In the experimental data presented in [23], the muscle fibre recovers faster; that is, it produces more force in the stimulation pulses after the tetanic stimulation. To mimic this behaviour, other states than the force output should be investigated to analyse which processes influence this recovery. This, however, was not the focus of this study. The key advantage of employing a biophysical model such as the one presented within this work, however, is that one can easily investigate other quantities than the force, for example, the intracellular $\mathrm{Ca}^{2+}$ concentration. 
Additionally to the investigated characteristics, that is, fatigue and contraction times, the model could be examined regarding the $\mathrm{Ca}^{2+}$ concentration progression as there exist more experimental data; see, for example, [22]. While the authors in [14] also determined the action potential conduction velocities on the fatigued and nonfatigued muscle fibres, muscle unit-specific experimental data are rare $[30,31]$. In [32], for example, only conduction velocities for slow-twitch and fast-twitch muscle are shown.

\section{Summary and Outlook}

This work presents an extension of the Shorten et al. model [14], which has been experimentally validated for slow-twitch and fast-twitch muscles, to investigate physiological properties of the different muscle units, which do exist in human skeletal muscles. To this end, the parameters of the computational model are determined by interpolating the parameter sets of the slow-twitch and fast-twitch muscles in different ways. The model revealed that different interpolations are required for different parameter groups to match experimental results. Using the resulting model, we identified one parameter group that predominantly affects fatigue, namely, the parvalbumin parameters, and two parameter groups that affect predominantly the contraction times, namely, the $\mathrm{Ca}^{2+}$ channel uptake rate and the cross-bridge parameters. Moreover, we found that the RyR current affected both contraction time and fatigue. Due to the fact that the model closely resembles the underlying physiological processes, it could potentially support the experimentalist investigating the mechanisms leading to peripheral muscle fatigue in the different muscle units. In turn, additional muscle unitspecific experimental data can improve mathematical models and hence contribute to an improved and validated mathematical model of the muscle units to investigate central fatigue using detailed chemoelectromechanical models of the entire neuromuscular system [21,33-36].

\section{Competing Interests}

The authors declare that they have no competing interests.

\section{Acknowledgments}

The research leading to these results has received funding from the European Research Council under the European Union's Seventh Framework Programme (FP/2007-2013)/ ERC Grant Agreement no. 306757 (LEAD).

\section{References}

[1] E. Henneman, G. Somjen, and D. O. Carpenter, "Excitability and inhibitability of motoneurons of different sizes," Journal of neurophysiology, vol. 28, no. 3, pp. 599-620, 1965.

[2] B. Bigland-Ritchie, "EMG and fatigue of human voluntary and stimulated contractions," in Human Muscle Fatigue: Physiological Mechanisms, pp. 130-156, John Wiley \& Sons, 1981.

[3] R. E. Burke, D. N. Levine, P. Tsairis, and F. E. Zajac, "Physiological types and histochemical profiles in motor units of the cat gastrocnemius," The Journal of Physiology, vol. 234, no. 3, pp. 733-748, 1973.

[4] E. M. Balog and R. H. Fitts, "Effects of fatiguing stimulation on intracellular $\mathrm{Na}+$ and $\mathrm{K}+$ in frog skeletal muscle," Journal of Applied Physiology, vol. 81, no. 2, pp. 679-685, 1996.

[5] N. A. Dimitrova and G. V. Dimitrov, "Interpretation of EMG changes with fatigue: facts, pitfalls, and fallacies," Journal of Electromyography and Kinesiology, vol. 13, no. 1, pp. 13-36, 2003.

[6] D. G. Allen, G. D. Lamb, and H. Westerblad, "Skeletal muscle fatigue: Cellular mechanisms," Physiological Reviews, vol. 88, no. 1, pp. 287-332, 2008.

[7] H. Westerblad, J. D. Bruton, and A. Katz, "Skeletal muscle: energy metabolism, fiber types, fatigue and adaptability," Experimental Cell Research, vol. 316, no. 18, pp. 3093-3099, 2010.

[8] P. J. Reiser, R. L. Moss, G. G. Giulian, and M. L. Greaser, "Shortening velocity in single fibers from adult rabbit soleus muscles is correlated with myosin heavy chain composition," The Journal of Biological Chemistry, vol. 260, no. 16, pp. 9077-9080, 1985.

[9] R. B. MacIntosh, F. P. Gardiner, and J. A. McComas, Skeletal Muscle: Form and Function, Human Kinetics, Champaign, Ill, USA, 2nd edition, 2006.

[10] C. J. Heckman and R. M. Enoka, "Motor unit," Comprehensive Physiology, vol. 2, no. 4, pp. 2629-2682, 2012.

[11] A. J. Fuglevand, D. A. Winter, and A. E. Patla, "Models of recruitment and rate coding organization in motor unit pools," Journal of Neurophysiology, vol. 70, no. 6, pp. 2470-2488, 1993.

[12] J. Duchateau and R. M. Enoka, "Human motor unit recordings: origins and insight into the integrated motor system," Brain Research, vol. 1409, pp. 42-61, 2011.

[13] R. Bottinelli and C. Reggiani, "Human skeletal muscle fibres: molecular and functional diversity," Progress in Biophysics and Molecular Biology, vol. 73, no. 2-4, pp. 195-262, 2000.

[14] P. R. Shorten, P. O'Callaghan, J. B. Davidson, and T. K. Soboleva, "A mathematical model of fatigue in skeletal muscle force contraction," Journal of Muscle Research and Cell Motility, vol. 28, no. 6, pp. 293-313, 2007.

[15] W. Wallinga, S. L. Meijer, M. J. Alberink, M. Vliek, E. D. Wienk, and D. L. Ypey, "Modelling action potentials and membrane currents of mammalian skeletal muscle fibres in coherence with potassium concentration changes in the T-tubular system," European Biophysics Journal, vol. 28, no. 4, pp. 317-329, 1999.

[16] E. Ríos, M. Karhanek, J. Ma, and A. González, “An allosteric model of the molecular interactions of excitation-contraction coupling in skeletal muscle," The Journal of General Physiology, vol. 102, no. 3, pp. 449-481, 1993.

[17] S. M. Baylor and S. Hollingworth, "Model of sarcomeric $\mathrm{Ca}^{2+}$ movements, including ATP $\mathrm{Ca}^{2+}$ binding and diffusion, during activation of frog skeletal muscle," The Journal of General Physiology, vol. 112, no. 3, pp. 297-316, 1998.

[18] M. V. Razumova, A. E. Bukatina, and K. B. Campbell, "Stiffnessdistortion sarcomere model for muscle simulation," Journal of Applied Physiology, vol. 87, no. 5, pp. 1861-1876, 1999.

[19] V. Augusto, C. R. Padovani, and G. E. R. Campos, "Skeletal muscle fiber types in C57BL6J mice," Brazilian Journal of Morphological Sciences, vol. 21, no. 2, pp. 89-94, 2004.

[20] T. Soukup, G. Zachařová, and V. Smerdu, "Fibre type composition of soleus and extensor digitorum longus muscles in normal female inbred Lewis rats," Acta Histochemica, vol. 104, no. 4, pp. 399-405, 2002.

[21] T. Heidlauf and O. Röhrle, "A multiscale chemo-electro-mechanical skeletal muscle model to analyze muscle contraction 
and force generation for different muscle fiber arrangements," Frontiers in Physiology, vol. 5, article 498, pp. 1-14, 2014.

[22] S. M. Baylor and S. Hollingworth, "Sarcoplasmic reticulum calcium release compared in slow-twitch and fast-twitch fibres of mouse muscle," The Journal of Physiology, vol. 551, no. 1, pp. 125138, 2003.

[23] J. Lannergren and H. Westerblad, "Force and membrane potential during and after fatiguing, continuous high-frequency stimulation of single Xenopus muscle fibres," Acta Physiologica Scandinavica, vol. 128, no. 3, pp. 359-368, 1986.

[24] K. B. Campbell, M. V. Razumova, R. D. Kirkpatrick, and B. K. Slinker, "Myofilament kinetics in isometric twitch dynamics," Annals of Biomedical Engineering, vol. 29, no. 5, pp. 384-405, 2001.

[25] F. Buchthal and H. Schmalbruch, "Spectrum of contraction times of different fibre bundles in the brachial biceps and triceps muscles of man," Nature, vol. 222, no. 5188, p. 89, 1969.

[26] M. van Cutsem, P. Feiereisen, J. Duchateau, and K. Hainaut, "Mechanical properties and behaviour of motor units in the tibialis anterior during voluntary contractions," Canadian Journal of Applied Physiology, vol. 22, no. 6, pp. 585-597, 1997.

[27] J. W. Fleshman, J. B. Munson, G. W. Sypert, and W. A. Friedman, "Rheobase, input resistance, and motor-unit type in medial gastrocnemius motoneurons in the cat," Journal of Neurophysiology, vol. 46, no. 6, pp. 1326-1338, 1981.

[28] D. G. Stuart, "Tetrapartite classification of motor units of cat tibialis posterior," Journal of Neurophysiology, vol. 44, no. 4, pp. 696-712, 1980.

[29] K. Kanda and K. Hashizume, "Factors causing difference in force output among motor units in the rat medial gastrocnemius muscle," The Journal of Physiology, vol. 448, no. 1, pp. 677-695, 1992.

[30] C. Vila-Chã, D. Falla, M. V. Correia, and D. Farina, "Adjustments in motor unit properties during fatiguing contractions after training," Medicine \& Science in Sports \& Exercise, vol. 44, no. 4, pp. 616-624, 2012.

[31] D. Farina, A. Holobar, M. Gazzoni, D. Zazula, R. Merletti, and R. M. Enoka, "Adjustments differ among low-threshold motor units during intermittent, isometric contractions," Journal of Neurophysiology, vol. 101, no. 1, pp. 350-359, 2009.

[32] C. Juel, "Muscle action potential propagation velocity changes during activity," Muscle \& Nerve, vol. 11, no. 7, pp. 714-719, 1988.

[33] T. Heidlauf, F. Negro, D. Farina, and O. Rohrle, "An integrated model of the neuromuscular system," in Proceedings of the 2013 6th International IEEE EMBS Conference on Neural Engineering (NER '13), pp. 227-230, IEEE, San Diego, Calif, USA, November 2013.

[34] T. Heidlauf and O. Röhrle, "Modeling the chemoelectromechanical behavior of skeletal muscle using the parallel opensource software library openCMISS," Computational and Mathematical Methods in Medicine, vol. 2013, Article ID 517287, 14 pages, 2013.

[35] M. Mordhorst, T. Heidlauf, and O. Röhrle, "Predicting electromyographic signals under realistic conditions using a multiscale chemo-electro-mechanical finite element model," Interface Focus, vol. 5, no. 2, Article ID 20140076, pp. 1-11, 2015.

[36] O. Röhrle, J. B. Davidson, and A. J. Pullan, "A physiologically based, multi-scale model of skeletal muscle structure and function," Frontiers in Physiology, vol. 3, article 358, pp. 1-14, 2012. 


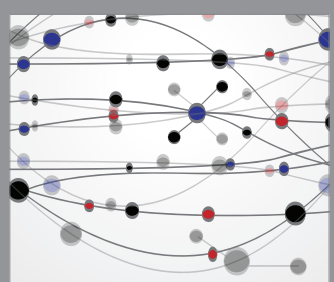

The Scientific World Journal
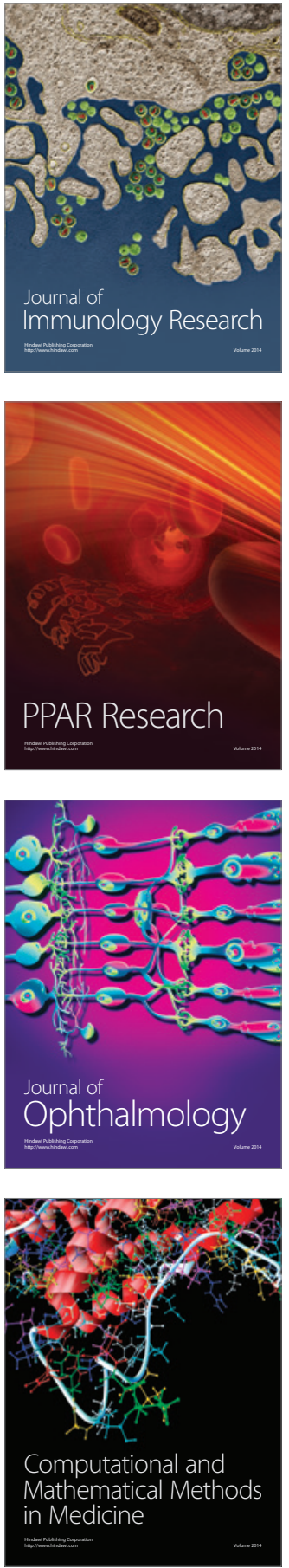

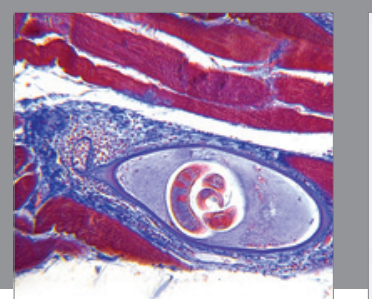

Gastroenterology Research and Practice

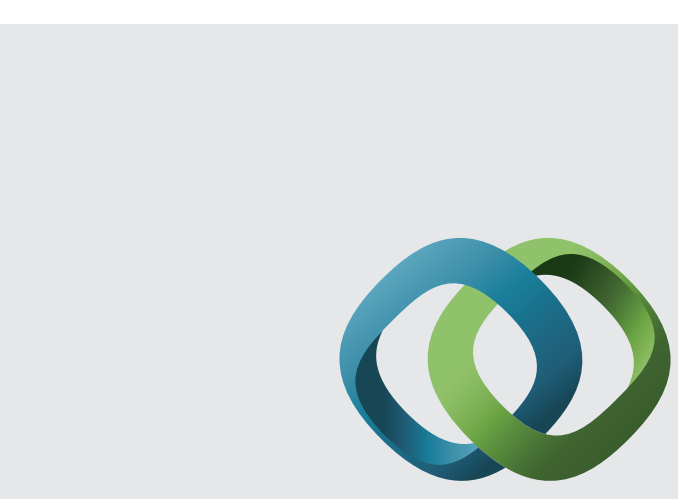

\section{Hindawi}

Submit your manuscripts at

http://www.hindawi.com
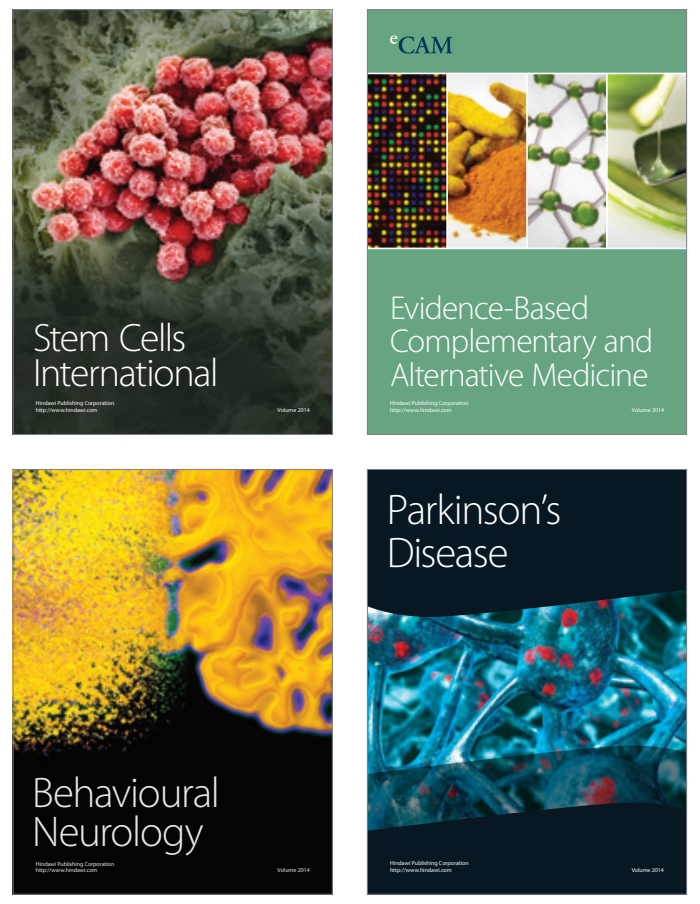
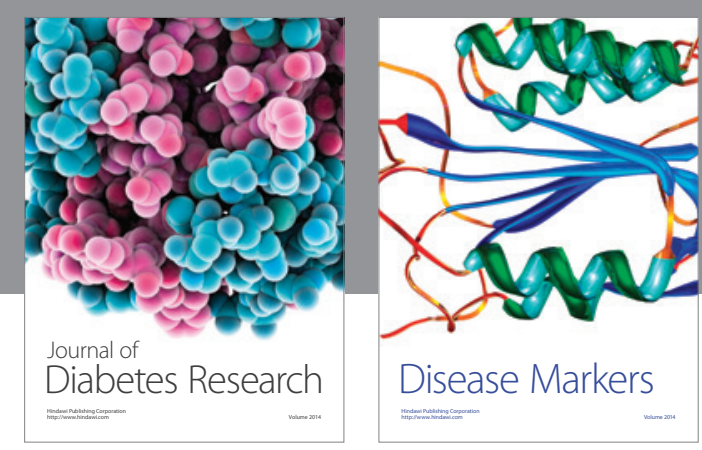

Disease Markers
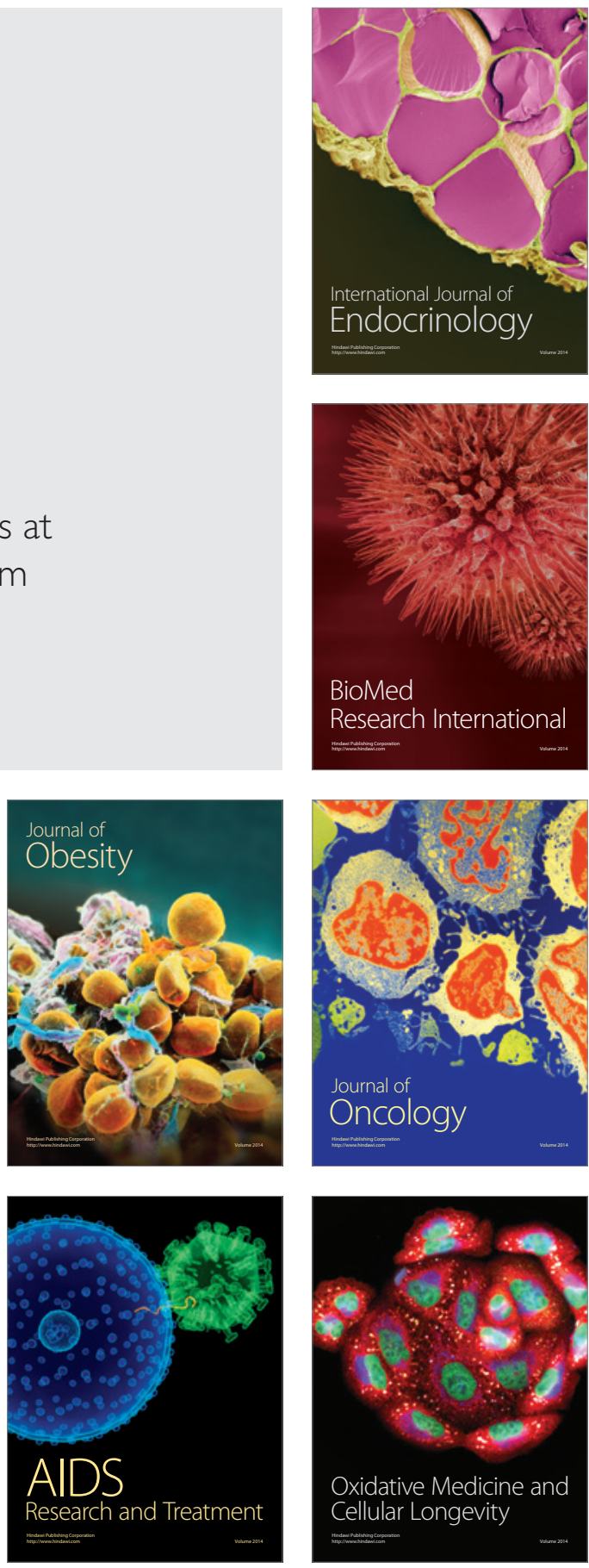